\title{
A DFT Study on (001) Thin Slabs of $\mathrm{SrTiO}_{3}$ and $\mathrm{BaTiO}_{3}$
}

\author{
T. MAHMOODI* \\ Department of Physics, Faculty of Sciences, Mashhad Branch, Islamic Azad University, Mashhad, Iran
}

(Received July 10, 2014)

\begin{abstract}
In this paper, we studied the relaxation of (001) surface of $\mathrm{BaTiO}_{3}$ and $\mathrm{SrTiO}_{3}$ slabs with two termination surfaces and with 3, 5, and 7 layers thickness for each cases, using density functional theory and generalized gradient approximation for exchange-correlation functional and pseudo potential method. We calculated the slab energy and the rate of expansion and contraction of the layers and compare them for different thicknesses. Band structure and density of states for these slabs and for $\mathrm{BaTiO}_{3}$ and $\mathrm{SrTiO}_{3}$ bulk were computed to find out the variation of band gap with respect to slab thickness. It is found that in comparison with bulk, in $\mathrm{TiO}_{2}$ slabs of both materials gap size decreases while in $\mathrm{SrO}$ and $\mathrm{BaO}$ slabs it increases.
\end{abstract}

DOI: 10.12693 /APhysPolA.127.1616

PACS: 31.15.A-, 31.15.E-, 82.45.Mp

\section{Introduction}

In recent decades ferroelectric materials have been grabbed great attention due to their technological and industrial applications. A class of these materials is perovskite oxides $\mathrm{ATiO}_{3}$ which display large piezoelectric, pyroelectric, and catalytic effects [1-6]. For these reasons thin films of $\mathrm{ATiO}_{3}$ perovskite ferroelectrics have been used significantly in microelectronic and piezoelectric devices. Moreover, these materials show non-linear optic response that makes them potentially suitable in electro-optical devices. Second harmonic generation has been reported in $\mathrm{BaTiO}_{3}$ thin films [4] and recently, the relationship between the structure of $\mathrm{BaTiO}_{3} / \mathrm{SrTiO}_{3}$ super lattices and the second-order nonlinear optical susceptibilities were studied $[7,8]$. Hence the surface structure of $\mathrm{ATiO}_{3}$ materials is very interesting. There are many theoretical researches on various surfaces of $\mathrm{ATiO}_{4}$ materials. The (001) surface of these materials contains neutral $\mathrm{AO}$ and $\mathrm{TiO}_{2}$ layers. This face is energetically stable, and has no dipole moment perpendicular to the surface. That is why the most of the experimental and computational investigations have been focused on (001) surface [9-13].

The main motivation of this paper is to study electronic structures and relaxation of $\mathrm{SrTiO}_{3}$ and $\mathrm{BaTiO}_{3}$ slabs with different thicknesses. The termination surface for these slabs is assumed along (001) surface. Our aim is to investigate the relaxation properties of (001) surface for such slabs with 3,5 , and 7 layers using density functional theory (DFT). Our calculations have been performed with Quantum-ESPRESSO [14] code and applying pseudo-potential method. We calculated the slab energy and the rate of expansion and contraction of the layers and compared them for different thicknesses quantitatively.

\footnotetext{
* e-mail: mahmoodi@mshdiau.ac.ir
}

\section{Calculation details}

$\mathrm{SrTiO}_{3}$ and $\mathrm{BaTiO}_{3}$ are dielectrics with perovskite crystal structure and $(p m 3 m)$ space group. This structure belongs to the generic structure of $\mathrm{ABO}_{3}$, in which on a cubic lattice an arrangement of $\mathrm{A}$ atoms on the corners, B atoms on the middle of the cubic faces and oxygen atoms on the center of the cube are located. Generally $\mathrm{B}$ atoms are transitional metals.

We have solved the self-consistent equations of KohnSham for these materials. We have done our calculation using Quantum-ESPRESSO code which is based on DFT and implementing pseudo-potentials. In this work we have used the Perdew-Burke-Ernzerhof (PBE) pseudo-potentials that are under the generalized gradient approximation (GGA). The converged values of necessary quantities have been obtained. In our calculations, a Monkhorst-Pack grid for $k$-point sampling as $(15 \times 15 \times 15)$ for $\mathrm{SrTiO}_{3}$ and as $(12 \times 12 \times 12)$ for $\mathrm{BaTiO}_{3}$ has been selected and a plane wave basis with a kinetic energy cutoff of $70 \mathrm{Ry}$ for $\mathrm{SrTiO}_{3}$ and $60 \mathrm{Ry}$ for $\mathrm{BaTiO}_{3}$ have been used. Using these quantities bulk equilibrium geometery was obtained using the analytical optimization approach as implemented in Quantum ESPRESSO. We calculated the equilibrium lattice constants and bulk moduli as $3.970 \AA$ and $190.9 \mathrm{GPa}$ for $\mathrm{SrTiO}_{3}$ and as $3.972 \AA$ and $222.8 \mathrm{GPa}$ for $\mathrm{BaTiO}_{3}$, respectively [15].

Slab calculations were done for both $\mathrm{SrTiO}_{3}$ and $\mathrm{BaTiO}_{3}$ slabs, using a super cell with odd number of layers $(3,5,7)$ and converged number of vacuum layers of $5,7,9$, respectively, and for two types of (001)surfaces, one type terminates to $\mathrm{TiO}_{2}$ and the other terminates to $\mathrm{SrO}(\mathrm{BaO})$ layer. Figure 1 shows a super cell for a 5 layers slab of both kinds for $\mathrm{BaTiO}_{3}$ which are the same as for $\mathrm{SrTiO}_{3}$. Optimized structure for each case was obtained after relaxation calculation.

\section{Surface relaxation}

Through calculating distances between the adjacent layers, we have obtained the rate of expansion or contrac- 

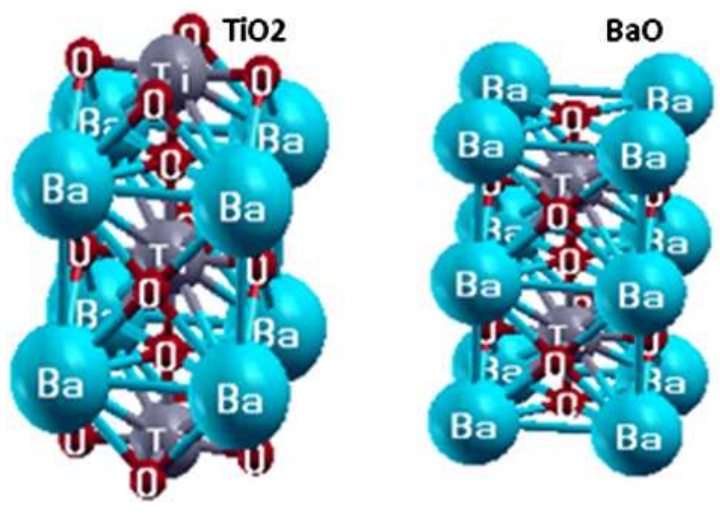

Fig. 1. The 5 layer slab super cells for two termination surface of $\mathrm{BaTiO}_{3}$.

tion of layers with respect to the bulk distances. The results are illustrated in Table I. As it is seen, except for 3 layer slabs with only two spacing, in other cases for both kinds of terminations, there is oscillating contractionexpansion and for the outermost two layers it is always contraction but the rate of contractions for $\mathrm{SrTiO}_{3}$ are larger than for $\mathrm{BaTiO}_{3}$. Second layers in both materials are expanded and in this case the rate of expansion for $\mathrm{SrTiO}_{3}$ are much less than for $\mathrm{BaTiO}_{3}$. Hence it can be deduced that the $\mathrm{SrTiO}_{3}$ slabs, generally, contract more than $\mathrm{BaTiO}_{3}$ slabs. Moreover, for both materials the rate of contraction in $\mathrm{TiO}_{2}$ layers is less than that in $\mathrm{Sro}(\mathrm{BaO})$ layers. It shows that the electronic charges distorted more on $\mathrm{SrO}(\mathrm{BaO})$ surfaces.

TABLE I

The rate of expansion or constraction of surface layers.

\begin{tabular}{c|c|c|c|c}
\hline \hline \multicolumn{2}{c|}{$n$} & $\frac{\Delta d_{12}}{d_{\text {bulk }} \times 100}$ & $\frac{\Delta d_{23}}{d_{\text {bulk }} \times 100}$ & $\frac{\Delta d_{34}}{d_{\text {bulk }} \times 100}$ \\
\hline \multirow{3}{*}{$\mathrm{SrO}$} & 3 & -6.09 & - & - \\
& 5 & -9.17 & +0.85 & - \\
& 7 & -9.72 & +0.95 & -2.26 \\
\hline \multirow{3}{*}{$\mathrm{TiO}_{2}$} & 3 & -4.48 & - & - \\
& 5 & -6.25 & +0.95 & - \\
& 7 & -6.09 & +1.66 & -1.96 \\
\hline \multirow{3}{*}{$\mathrm{BaO}$} & 3 & -3.27 & - & - \\
& 5 & -6.74 & +329 & - \\
& 7 & -7.19 & +5.18 & -1.99 \\
\hline \multirow{2}{*}{$\mathrm{TiO}_{2}$} & 3 & -1.81 & - & - \\
& 5 & -3.62 & +2.16 & - \\
& 7 & -3.27 & +3.72 & -0.25
\end{tabular}

Furthermore, we obtained slab energy for each thickness. This energy is the necessary energy for creating a slab with defined thickness, and is determined as Eq. (1):

$$
E_{\mathrm{s}}=\frac{1}{4}\left[E_{\mathrm{AO}}^{\mathrm{relax}}+E_{\mathrm{TiO}_{2}}^{\mathrm{relax}}-n E_{\mathrm{bulk}}\right] \text {, }
$$

in which $E_{\mathrm{s}}$ is the slab energy, $n$ is the number of layers, $E_{\mathrm{AO}}^{\mathrm{relax}}$ and $E_{\mathrm{TiO}_{2}}^{\mathrm{relax}}$ are total energies of two kinds of relaxed slabs where in $E_{\mathrm{AO}}^{\text {relax }}$, A stands for $\mathrm{Sr}$ or Ba. Calculated slab energy for slabs with 3,5 , and 7 layers, re- spectively, are $(1.09,0.99,0.95) \mathrm{eV}$ for $\mathrm{SrTiO}_{3}$ and $(0.95$, 0.86, 0.82) eV for $\mathrm{BaTiO}_{3}$. These results reveal that in both cases thicker slabs need less energy to form. Besides it is seen that $\mathrm{SrTiO}_{3}$ slabs in comparison with $\mathrm{BaTiO}_{3}$ slabs need more energy to create, in complete agreement with the above results of more contraction of both kinds of $\mathrm{SrTiO}_{3}$ slabs.

\section{Electronic structure}

$\mathrm{SrTiO}_{3}$ and $\mathrm{BaTiO}_{3}$ are semiconductors with experimental bulk gap of $3.25 \mathrm{eV}$ [16] and $3.2 \mathrm{eV}$ [16], respectively. We calculated electronic structures for $\mathrm{SrTiO}_{3}$ and $\mathrm{BaTiO}_{3}$ bulk and all kinds of the slabs. Band structure, DOS and PDOS for bulk $\mathrm{SrTiO}_{3}$ and $\mathrm{BaTiO}_{3}$ are shown in Fig. 2 and Fig. 3, respectively.

A comparison between these two bands show that there is a flat area for HOMO in both crystal which occurs between points $X$ and $M$. Besides $\Gamma$ point in $\mathrm{BaTiO}_{3}$ is also a HOMO point but not in $\mathrm{SrTiO}_{3}$. This subject causes that the magnitude of direct band gap $\Gamma-\Gamma$ in $\mathrm{BaTiO}_{3}$ was less than in $\mathrm{SrTiO}_{3}$. The results for DOS and PDOS are plotted in the same Figure. These curves verify the smallest band gap for both materials and show that the valance band width for BaTiO3 is $4.5 \mathrm{eV}$ and for $\mathrm{SrTiO}_{3}$ is $4.6 \mathrm{eV}$. Besides the PDOS plot depicts that the valance band mainly consists of the $2 p$ states of $\mathrm{O}$ near the Fermi energy edge, and $d$-orbital states of $\mathrm{Ti}$ predominantly plays a main role for the conduction band above the Fermi level.
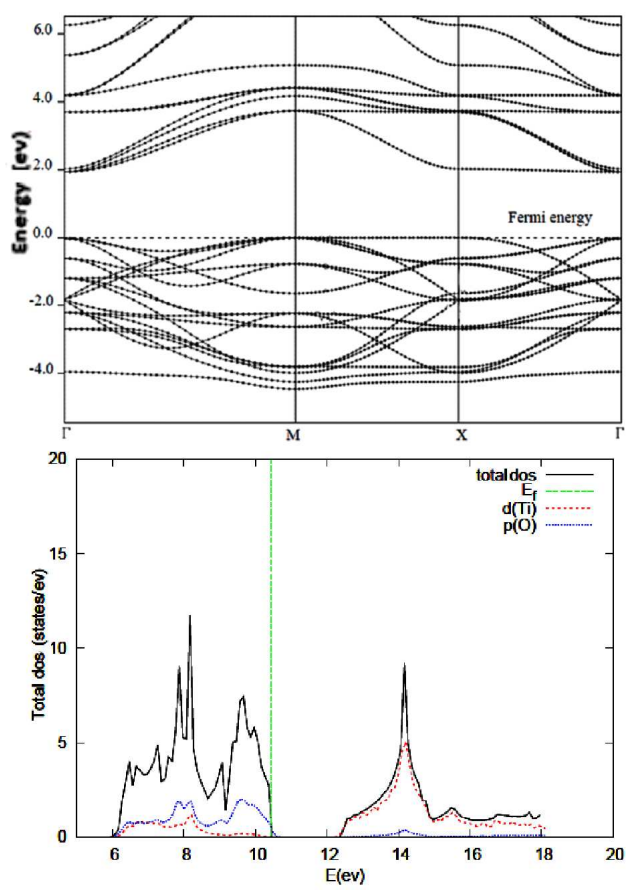

Fig. 2. Band structure and DOS for bulk $\mathrm{BaTiO}_{3}$.

At the next step we studied the effect of thickness on the electronic structure of $\mathrm{SrTiO}_{3}$ and $\mathrm{BaTiO}_{3}$ slabs with two terminations of $\mathrm{AO}(\mathrm{A}=\mathrm{Sr}, \mathrm{Ba})$ and $\mathrm{TiO}_{2}$. Band 

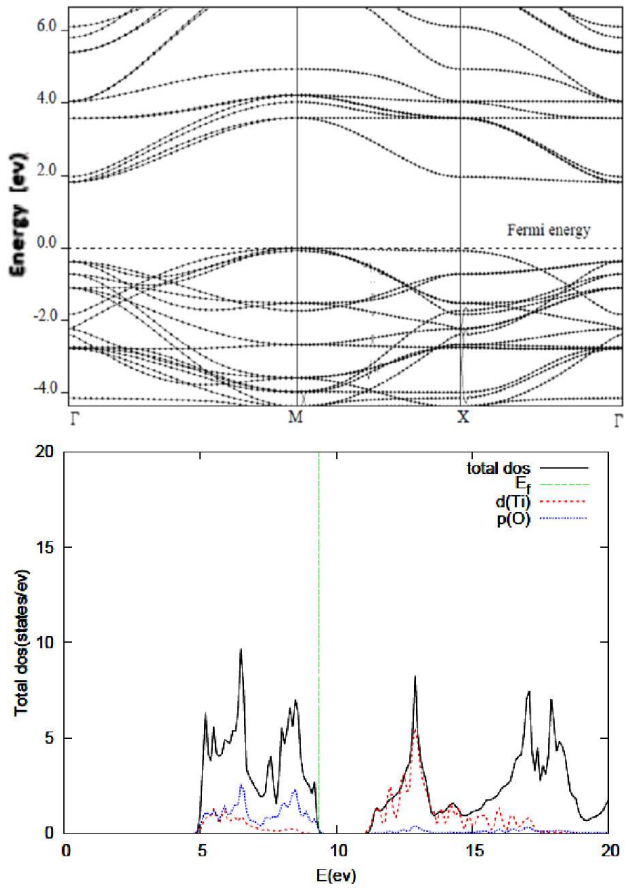

Fig. 3. Band structure and DOS for bulk $\mathrm{SrTiO}_{3}$.

structure and total DOS of theses slabs are presented in Figs. 4-7. As it is seen, the same as band structures of bulk, there is a flat area near gap in both crystals of $\mathrm{SrTiO}_{3}$ and $\mathrm{BaTiO}_{3}$ which in $\mathrm{TiO}_{2}$ terminated slabs of two crystals occur at the bottom of conduction band between $\Gamma$ and $X$ points, while in $\mathrm{SrO}$ and $\mathrm{BaO}$ terminated slabs occur at the top of valence band between $M$ and $X$ points. This flat area causes two or three indirect gaps with nearly the same magnitude.
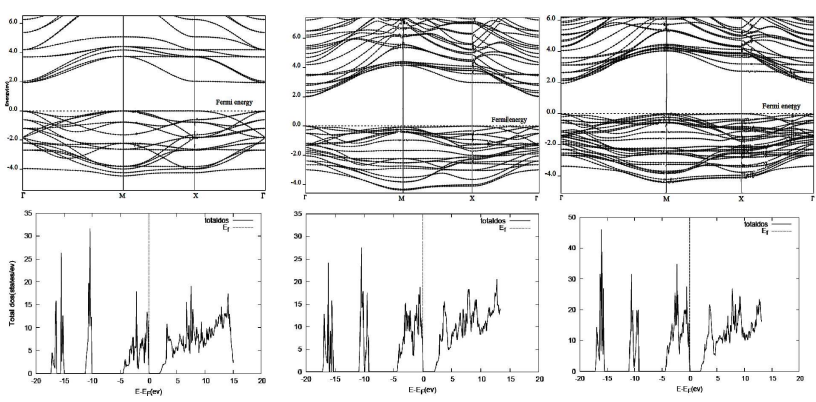

Fig. 4. Band structure, DOS and PDOS for $\mathrm{BaO}$ slabs of $\mathrm{BaTiO}_{3}$ for $n=3,5,7$ respectivly from left to right.

Calculated direct and indirect band gaps are summarized in Table II. In SrO slabs, the smallest gap is $X-\Gamma$ type and it is slightly more than the bulk gap magnitude with $1.82 \mathrm{eV}$. In $\mathrm{BaO}$ slabs, direct gap $\Gamma-\Gamma$ and indirect gaps of $M-\Gamma$ and $X-\Gamma$ with nearly the same size are the smallest gaps, which are similar to bulk $\mathrm{BaTiO}_{3}$. In both kinds of $\mathrm{TiO}_{2}$ slabs, $M-\Gamma$ and $M-X$ gaps are the smallest one which indicate that in $\mathrm{TiO}_{2}$ slabs gap size decreases with respect to bulk, while in AO slabs it increases. Finally it should be noted that in $\mathrm{SrO}$ and
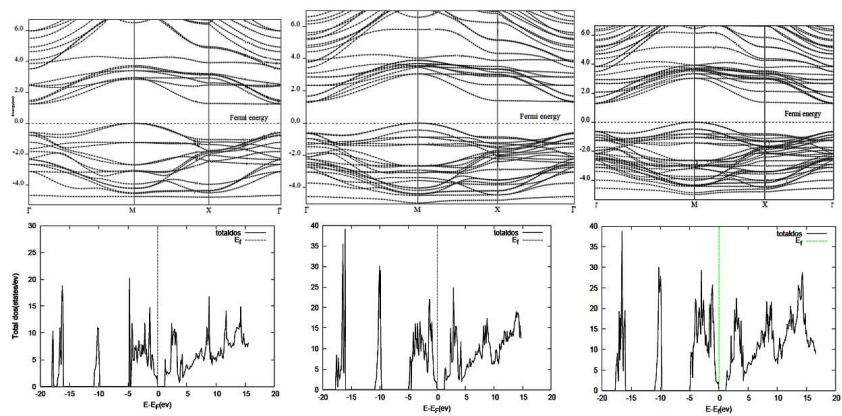

Fig. 5. As Fig. 4 for $\mathrm{TiO}_{2}$ slabs.

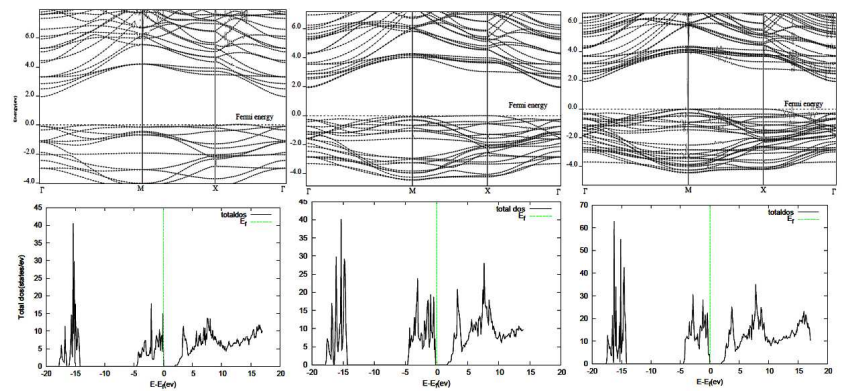

Fig. 6. Band structure, DOS and PDOS for Sr slabs of $\mathrm{SrTiO}_{3}$ for $n=3,5,7$ respectivly from left to right.

$\mathrm{BaO}$ slabs, as the slab thickness is increasing, gap size is decreasing to reach the magnitude of bulk.

On the other hand in $\mathrm{TiO}_{2}^{\mathrm{SrTiO}_{3}}\left(\mathrm{TiO}_{2}\right.$ terminated slabs of $\mathrm{SrTiO}_{3}$ ) it seems that there are oscillating behaviors that may refer to quantum size effects while in $\mathrm{TiO}_{2}^{\mathrm{BaTiO}_{3}}$ (analogously) all types of gaps do not show any regular behavior.

We have also plotted the DOS curves for all kinds of $\mathrm{SrTiO}_{3}$ and $\mathrm{BaTiO}_{3}$ slabs in Figs. 4-7. As it can be seen, all the features of slab-DOS were found to be almost unaltered in comparison to bulk states, except that the energy gap is less than the bulk in all cases. Near the conduction band edge, in both $\mathrm{SrTiO}_{5}$ and $\mathrm{BaTiO}_{3}$ slabs, density of states is different from each other for $\mathrm{AO}$ and $\mathrm{TiO}_{2}$ termination slabs slightly due to the change of electron distribution. As in AO slabs it starts to rise with a gentle slope, while in $\mathrm{TiO}_{2}$ slabs it raises almost abruptly. Hence, $\mathrm{TiO}_{2}$ slabs can induce a better electron excitation probability.
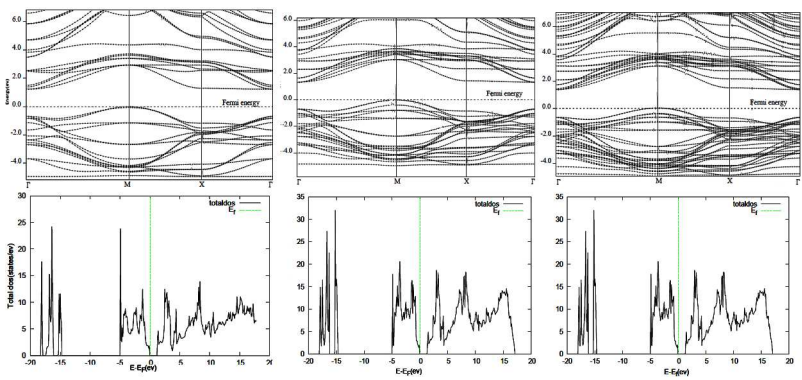

Fig. 7. As Fig. 6 for $\mathrm{TiO}_{2}$ slabs. 
TABLE II

Direct and indirect band gaps in eV.

\begin{tabular}{c|c|c|c|c|c|c}
\hline \hline & & \multicolumn{5}{|c}{ Gap type } \\
\cline { 3 - 7 } & $n$ & $M-\Gamma$ & $X-\Gamma$ & $M-X$ & $X-X$ & $\Gamma-\Gamma$ \\
\hline \multirow{3}{*}{$\mathrm{SrO}$} & 3 & 2.09 & 1.99 & 3.61 & 3.51 & 2.03 \\
& 5 & 1.99 & 1.93 & 3.10 & 3.03 & 2.17 \\
& 7 & 1.91 & 1.90 & 2.80 & 2.79 & 2.18 \\
\hline \multirow{2}{*}{$\mathrm{TiO}_{2}$} & 3 & 1.22 & 2.40 & 1.30 & 2.47 & 1.92 \\
& 5 & 0.91 & 1.03 & 1.04 & 2.27 & 1.99 \\
& 7 & 1.33 & 2.06 & 1.48 & 2.21 & 2.02 \\
\hline $\mathrm{SrTiO}_{3-\text { bulk }}$ & & 1.82 & 1.90 & 1.97 & 2.04 & 2.18 \\
\hline \multirow{3}{*}{$\mathrm{BaO}^{*}$} & 3 & 2.01 & 2.08 & 3.41 & 3.48 & 1.96 \\
& 5 & 2.08 & 2.01 & 3.05 & 2.98 & 2.02 \\
$\mathrm{TiO}_{2}$ & 7 & 1.94 & 1.96 & 2.72 & 2.73 & 1.99 \\
\hline & 3 & 1.25 & 2.31 & 1.28 & 2.34 & 1.84 \\
& 5 & 1.27 & 2.13 & 1.39 & 2.25 & 1.87 \\
& 7 & 1.27 & 2.07 & 1.37 & 2.16 & 1.90 \\
\hline $\mathrm{BaTiO}_{3-\text { bulk }}$ & & 1.93 & 1.93 & 2.03 & 2.03 & 1.96
\end{tabular}

\section{Conclusion}

In this paper, the electronic structure of thin $\mathrm{SrTiO}_{3}$ and $\mathrm{BaTiO}_{3}$ slabs are studied using QuantumESPRESSO code in the framework of DFT. We have calculated equilibrium geometry, slab energy, band structure and density of states for bulk $\mathrm{SrTiO}_{3}$ and $\mathrm{BaTiO}_{3}$ and thin slabs with two kinds of termination surfaces with 3,5 , and 7 layers thickness in each kind. Our results show that in all slabs there is an oscillating contractionexpansion and slab energy is more in thinner slabs. Moreover, thin slabs of $\mathrm{SrTiO}_{3}$ and $\mathrm{BaTiO}_{3}$ remain semiconducting but the band gap of $\mathrm{TiO}_{2}$ slabs decreases with respect to the bulk band gap, while in $\mathrm{SrO}$ slabs it increases. There is also a difference between DOS for two kinds of slabs near the conduction band edge that can affect on electron excitation.

\section{References}

[1] A. Yoffe, H. Cohen, V. Shelukhin, Zh. Tekhn. Fiz. Technical Physics 57, 134 (2012).

[2] K.-I. Park, S. Xu, Y. Liu, G.-T. Hwang, S.-J.L. Kang, Z.L. Wang, Nano Letters. 10, 4939 (2010.

[3] A. Erba, K.E. El-Kelany, M. Ferrero, I. Baraille, M. Rérat, Phys. Rev. B 88, 035102 (2013).

[4] I. Popescu, I. Săndulescu, 'A. Rédey, I.-C. Marcu, Catal. Lett. 141, 145 (2011).

[5] Y. Cui, J. Briscoe, S. Dunn, Chem. Mater. 25, 4215 (2013).

[6] J. Wu, M. Muruganandham, L. Chang, G. Lee, V. Batalova, G. Mokrousov, Ozone: Sc.ESEng. 33, 74 (2011).

[7] H. Lu, L. Wills, B. Wessels, W. Lin, T. Zhang, G. Wong, D. Neumayer, T. Marks, Appl. Phys. Lett. 62, 1314 (1993).

[8] T. Zhao, F. Chen, H. Lü, G. Yang, Z. Chen, Sci. China Series A Math. 43, 760 (2012).

[9] R. Eglitis, Int. J. Modern Phys. B , 28 (2014).

[10] A. Barbier, C. Mocuta, D. Stanescu, P. Jegou, N. Jedrecy, H. Magnan, J. Appl. Phys. 112, 114116 (2012).

[11] R. Eglitis, Phase Trans. 86, 1115 (2013).

[12] G.-Z. Zhu, G. Radtke, G.A. Botton, Nature 490, 384 (2012).

[13] Z.-Q. Li, J.-L. Zhu, C. Wu, Z. Tang, Y. Kawazoe, Phys. Rev. B 58, 8075 (1998).

[14] S. Baroni, A. Dal Corso, S. de Gironcoli, P. Giannozzi, C. Cavazzoni, G. Ballabio, Quantum ESPRESSO: open-source package for research in electronic structure, simulation, and optimization, www.quantumespresso.org.

[15] K.H. Hellwege, A.M. Hellwege, Ferroelectrics and Related Substances, New Series, Group III, Eds.: K.H. Hellwege, A.M. Hellwege, Springer-Verlag, Berlin 1969.

[16] K. van Benthem, C. Elsässer, R. French, J. Appl. Phys. 90, 6156 (2001).

[17] S.H. Wemple, Phys. Rev. B 2, 2679 (1970). 